\title{
CT Guided Bone Marrow Aspiration and Core Biopsy
}

\author{
Carlos M. Badiola ${ }^{1}$, Folco Scappaticci ${ }^{2}$, Driola Brahaj ${ }^{3}$ \\ ${ }^{1}$ Department of Radiology, Bristol Hospital, Bristol, Connecticut, USA \\ ${ }^{2}$ Department of Radiology, University of Connecticut Health Center, Farmington, Connecticut, USA \\ ${ }^{3}$ Department of Medical Oncology, Bristol Hospital, Bristol, Connecticut, USA \\ Email: carlioso@yahoo.com
}

Received April 29, 2012; revised May 26, 2012; accepted June 1, 2012

\begin{abstract}
Hemorrhagic complications related to bone marrow aspiration and trephine biopsy are uncommon. Risk factors include thrombocytopenia, myeloproliferative disorders, concurrent use of anticoagulants, and obesity. Here we describe our technique for bone marrow aspiration and biopsy of the posterior iliac crest using CT guidance. Our technique ensures needle passage only through subcutaneous fat and bone, avoiding muscle and neurovascular structures, as well as controlled and precise needle advancement within bone. This technique should be considered for use in patients that are at increased risk for procedure related complications, particularly obese patients, where surface anatomical landmarks may prove unreliable.
\end{abstract}

Keywords: Interventional Oncology; Biopsy; CT; Skeletal Interventions; Bone

\section{Introduction}

Severe hemorrhagic complications related to bone marrow aspiration and trephine biopsy are rare [1]. Risk factors include thrombocytopenia, myeloproliferative disorders, concurrent use of anticoagulants, and obesity [1, 2]. In obese patients, surface anatomic landmarks may be difficult to ascertain; thereby increasing the risk of injury to adjacent vascular or neural structures during bone marrow biopsy. Our purpose is to describe our technique for bone marrow aspiration and biopsy of the posterior iliac crest using CT guidance. The use of CT guidance ensures passage of the biopsy needle only through subcutaneous fat and bone, completely avoiding adjacent muscle and nearby neurovascular structures. This technique should be considered for use in patients that are at increased risk for procedure related complications, and in particular, obese patients, where surface anatomical landmarks may prove unreliable.

\section{Materials and Methods}

A total of 31 adult patients were referred to our interventional radiology service for CTguided bone marrow aspiration and biopsy between June 1, 2009 and April 30, 2012. These patients were considered at increased risk for procedure related complications due to obesity or coagulopathy. The majority of the referrals were related to the inability of the referring oncologist to confidently ascertain surface anatomic landmarks due to obesity. All of the procedures were performed for the evaluation of hematological disorders.

After obtaining informed consent, the patient is placed prone on the CT table and preliminary sections obtained through the posterior iliac crests. The biopsy procedures were performed with the aid of CT fluoroscopy. All patients received conscious sedation with midazolam or a combination of midazolam and fentanyl. The site selected for biopsy was the mid posterior iliac crest. The left side was most commonly chosen based on the layout of our CT suite. The selection of the site for biopsy ensured passage only though subcutaneous fat and bone, with avoidance of the adjacent gluteus and paraspinal musculature (Figure 1).

After selecting the site for biopsy based on the initial CT images, the skin overlying the area is cleansed with betadine and draped in sterile manner. The selected needle trajectory is anesthetized with $1 \%$ lidocaine, includeing injection of anesthetic into the bone surface. A small incision is then made with a surgical scalpel to allow for passage of the biopsy needle. We use an 11 gauge Jamshidi bone marrow biopsy needle (Cardinal Health, McGaw Park, Illinois). With the aid of CT fluoroscopy, the needle is advanced into the bone using gentle taps with a mallet in a manner similar to our technique for advancing a needle into a vertebra during vertebroplasty. The use of a mallet ensures controlled advancement of the biopsy needle into the bone, further lessening the risk of complications by avoiding uncontrolled advancement of the needle beyond the intended site of biopsy or aspiration. 


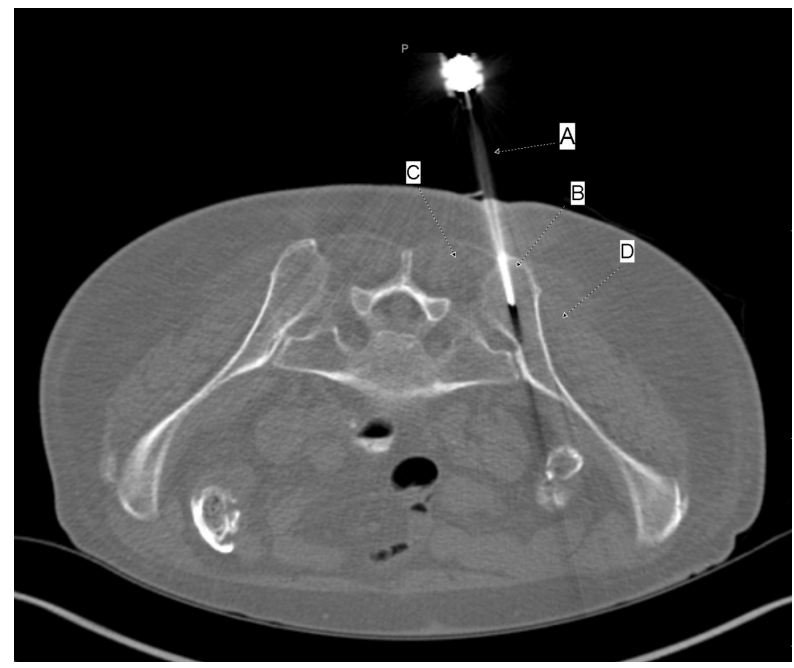

Figure 1. CT image shows an 11 gauge Jamshidi needle (A) that has been advanced into the marrow space of the left posterior iliac crest. The needle traverses only subcutaneous fat and bone (B), avoiding the adjacent muscles (C and D) and the pelvic neurovascular structures.

After placement of the needle into the marrow space of the posterior iliac crest, the inner stylet is removed and marrow aspiration is performed. After marrow aspiration, the needle is pulled back to the bone surface entry site and redirected at a slightly different angle than that used for aspiration. The needle and inner stylet are again advanced with gentle taps using a mallet until the bone is securely engaged, usually no more than a few millimeters. The inner stylet in then removed and the needle advanced a further $2 \mathrm{~cm}$ into the bone with the mallet. The needle is then rotated and gently rocked at different angles to ensure an adequate core specimen. The needle is then removed completely and the core specimen pushed out from the needle with the use of a blunt pushing cannula.

All of the aspiration and trephine specimens where diagnostic and no procedure related complications where observed.

\section{Discussion}

Bone marrow aspiration and biopsy of the posterior iliac crest using surface anatomical landmarks is a safe and effective procedure that is most commonly performed for the evaluation of hematologic disorders. In experienced hands, the complication rate is very low [1]. The most feared complication is life threatening hemorrhage. Death has been reported due to severe hemorrhagic complications [1]. Risk factors for hemorrhagic complications include thrombocytopenia, myeloproliferative disorders, concurrent use of anticoagulants, and obesity [1,2]. In obese patients, surface anatomic landmarks are more difficult to ascertain and the needle trajectory is longer, in- creasing the risk of improper needle placement.

Severe hemorrhagic complications related to bone marrow aspiration and biopsy are due to improper needle placement and laceration of adjacent arteries, such as the superior gluteal artery or small muscular arteries, or the inadvertent passage of the needle through the targeted bone and into the retroperitoneum [3-5]. These complications can be avoided with the use of CT guidance. CT guidance ensures proper needle placement into the bone, as well as the avoidance of all anatomic structures other than subcutaneous fat and bone. In addition, we believe that the use of a mallet for advancing the needle into the bone, in a manner commonly used for percutaneous vertebroplasty, increases the safety of the procedure by ensuring controlled and precise advancement of the biopsy needle, lessening the risk of inadvertent passage into the retroperitoneum [6].

Drawbacks to the use of CT guidance include added costs and associated radiation. Given the established low complication rate of bone marrow aspiration and biopsy when performed by experienced operators, the routine use of CT guidance is difficult to justify. Nevertheless, in patients at increased risk of bleeding complications due to a coagulopathy, and increasingly prevalent in current clinical practice, in obese patients where surface anatomic landmarks may prove unreliable, bone marrow aspiration and biopsy using CT guidance may help avoid the risk of hemorrhagic complications.

\section{REFERENCES}

[1] B. J. Bain, "Bone Marrow Biopsy Morbidity and Mortality,” British Journal of Haematology, Vol. 121, No. 6, 2003, pp. 949-951. doi:10.1046/j.1365-2141.2003.04329.x

[2] S. Malempati, S. Joshi, S. Lai, et al., "Bone Marrow Aspiration and Biopsy," New England Journal of Medicine, Vol. 361, 2009, p. e28.

[3] R. M. Lowenthal, B. V. Taylor, R. Jones and A. Beasly, "Severe Persistent Sciatic Pain and Weakness Due to a Gluteal Artery Pseudoaneurysm as a Complication of Bone Marrow Biopsy,” Journal of Clinical Neuroscience, Vol. 13, No. 3, 2006, pp. 384-385. doi:10.1016/j.jocn.2005.03.027

[4] P. Salem, M. K. Wolverson, H. J. Reimers and G. C. Kudva, "Complications of Bone Marrow Biopsy," British Journal of Haematology, Vol. 121, No. 6, 2003, p. 821. doi:10.1046/j.1365-2141.2003.04328.x

[5] M. Luoni, et al., "Retroperitoneal Hemorrhage Following Bone Marrow Biopsy,” Haematologica, Vol. 79, No. 1, 1994, pp. 70-72.

[6] J. M. Mathis and W. Wong, "Percutaneous Vertebroplasty, Technical Considerations,” Journal of Vascular and Interventional Radiology, Vol. 14, No. 8, 2003, pp. 953-960. doi:10.1097/01.RVI.0000083255.29749.A8 